Marcelo Wanini Fernandes S. de Souza

\title{
O Desempenho dos Setores da Indústria Nacional, Formados pelas Empresas de Capital Aberto, Diante do Nível da Atividade Econômica do Brasil e do Mundo
}

\section{DISSERTAÇÃO DE MESTRADO}

\author{
DEPARTAMENTO DE ADMINISTRAÇÃO \\ Programa de Pós-Graduação em Administração \\ de Empresas
}




\section{Pontifícia Universidade Católica $_{\text {a }}$

Marcelo Wanini Fernandes S. de Souza

O Desempenho dos Setores da Indústria Nacional, Formados pelas Empresas de Capital Aberto, Diante do Nível da Atividade Econômica do Brasil e do Mundo

Dissertação de Mestrado

Dissertação apresentada como requisito parcial para obtenção do grau de Mestre pelo Programa de Pósgraduação em Administração de Empresas do Departamento de Administração da PUC-Rio.

Orientador: Prof. Dr. Walter Lee Ness, Jr. 


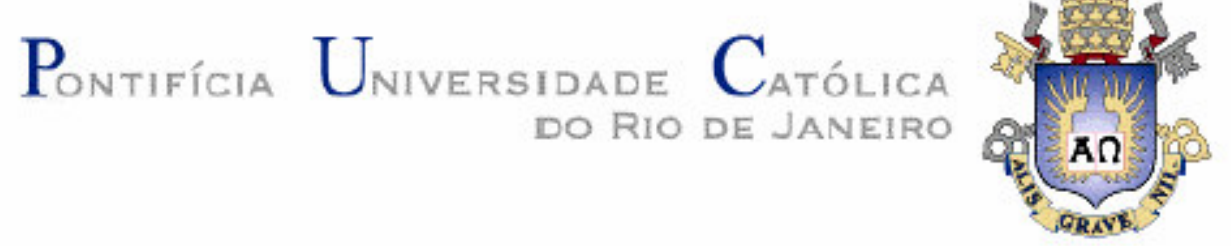

Marcelo Wanini Fernandes S. de Souza

O Desempenho dos Setores da Indústria Nacional, Formados pelas Empresas de Capital Aberto, Diante do Nível da Atividade Econômica do Brasil e do Mundo

Dissertação apresentada como requisito parcial para obtenção do grau de Mestre pelo Programa de PósGraduação em Administração de Empresas do Departamento de Administração do Centro de Ciências Sociais da PUC-Rio. Aprovada pela Comissão Examinadora abaixo assinada

Prof. Walter Lee Ness, Jr.

Orientador

Departamento de Administração, PUC-Rio

Prof. Marcelo Cabus Klotzle

Departamento de Administração, PUC-Rio

Prof. Claudio Roberto Contador

Funenseg

Profa. Zélia M. de Lossio e Seiblitz

Coordenadora Setorial do Centro de Ciências

Sociais da PUC-Rio

Rio de Janeiro, 11 de agosto de 2003 
Todos os direitos reservados. É proibida a reprodução total ou parcial do trabalho sem autorização da universidade, do autor e do orientador.

\section{Marcelo Wanini Fernandes Soares de Souza}

Graduou-se em Ciências Contábeis pela Universidade Federal do Rio de Janeiro em 1997. Além do mestrado, possui pós-graduação em Administração Financeira pela Fundação Getúlio Vargas, concluída em 1998 e é Tecnólogo em Telecomunicações pela Universidade Estácio de Sá, curso de graduação concluído em 1991. No âmbito profissional atuou nas áreas Contábil, Orçamento e Custos e Planejamento Financeiro da Latasa S.A. Foi um dos líderes das equipes dos projetos de implementação do Sistema Integrado de Gestão (ERP) e da metodologia Six Sigma da mesma empresa.

Ficha Catalográfica

Souza, Marcelo Wanini Fernandes Soares de

O Desempenho dos Setores da Indústria Nacional, Formados pelas Empresas de Capital Aberto, Diante do Nível da Atividade Econômica do Brasil e do Mundo/ Marcelo Wanini Fernandes Soares de Souza; orientador: Walter Lee Ness, Jr. - Rio de Janeiro : PUC, Departamento de Administração, 2003.

161 f. : il. ; $30 \mathrm{~cm}$

Dissertação (mestrado) - Pontifícia Universidade Católica do Rio de Janeiro, Departamento de Administração.

Inclui referências bibliográficas e anexos.

1. Administração - Teses. 2. Desempenho dos Setores da Indústria Nacional. 3. Nível de Atividade Econômica. 4. Elasticidade. 5. Regressão. 6. Modelos de Previsão. 7. PIB. 8. GDP. 9. Níveis de Lucro. I. Ness Jr., Walter Lee. II. Pontifícia Universidade Católica do Rio de Janeiro. Departamento de Administração. III. Título. 
À minha esposa, pela confiança e apoio incondicionais, fundamentais para a conclusão desta jornada. 


\section{Agradecimentos}

Aos meus pais, Wanini e Marly, pelo carinho, pelo apoio irrestrito e pelos sacrifícios que fizeram para proporcionar sempre a melhor educação, mostrando a importância da boa formação para a realização pessoal e profissional;

À minha família, pela incentivo sempre constante e pela crença em meu sucesso;

Ao professor Ness, pela paciência, pelo interesse em ajudar, pela competência técnica demonstrada ao longo do curso e pelo incentivo e apoio para a conclusão deste trabalho;

Ao professor Marcelo, pelas oportunas e valiosas contribuições apresentadas no processo de revisão deste estudo;

Ao professor Contador, pelas referências que nortearam direta e indiretamente este trabalho;

Aos amigos da turma de mestrado de 2001, por proporcionarem um ambiente sempre amistoso e cooperativo, tornando mais agradável nosso convívio ao longo destes anos;

Aos professores e funcionários da PUC, pela dedicação e pelo profissionalismo demonstrados nestes anos de convívio;

À minha esposa Mariana, que soube compreender as dificuldades enfrentadas durante o curso, abrindo mão de muitos fins-de-semana para que eu pudesse atingir meus objetivos. Com ela divido igualmente minha conquista. 


\section{Resumo}

Souza, Marcelo Wanini Fernandes Soares de; Ness Jr., Walter Lee (Orientador). O Desempenho dos Setores da Indústria Nacional, Formados pelas Empresas de Capital Aberto, Diante do Nível da Atividade Econômica do Brasil e do Mundo. Rio de Janeiro, 2003. 161p. Dissertação de Mestrado - Departamento de Administração, Pontifícia Universidade Católica do Rio de Janeiro.

Tendo como principal objetivo a avaliação do desempenho dos setores da indústria nacional, formados pelas empresas de capital aberto, diante do nível da atividade econômica do Brasil e do mundo, o presente trabalho tenta contribuir para a criação de modelos de previsão de forma a auxiliar administradores de empresas e acionistas em seus precessos decisórios. Foram exploradas as relações entre os diversos níveis de lucros representados pela Receita Operacional Líquida, Lucro Bruto, Lucro Operacional Próprio, Lucro Operacional e Lucro Líquido obtidos pelos setores da indústria nacional formados pelas empresas de capital aberto listadas na Bovespa e o nível da atividade econômica, representado pelo PIB nacional e mundial no período compreendido entre janeiro de 1996 a dezembro de 2002 em bases trimestrais. Na primeira parte são analisadas as elasticidades dos diversos níveis de lucro dos setores avaliados em função das variações do PIB. Na segunda parte foi analisado o grau de correlação entre o PIB mundial e o desempenho dos setores. Foi então comprovada a existência de colinearidade significativa entre o PIB mundial e o PIB nacional, não permitindo avaliar o desempenho dos setores ao se utilizar estas variáveis simultaneamente. Na última parte procurou-se incluir outras variáveis macroeconômicas, obtendo-se maior poder explanatório para o desempenho dos setores diante das variações do nível da atividade econômica nacional. Dado o nível de significância estatística obtido nos testes, comprova-se o potencial em utilizar estas relações em modelos preditivos.

\section{Palavras-chave}

Desempenho dos Setores da Indústria Nacional; Nível de Atividade Econômica; Elasticidade; Regressão; Modelos de Previsão; PIB; GDP; Níveis de Lucro. 


\section{Abstract}

Souza, Marcelo Wanini Fernandes Soares de; Ness, Walter Lee (Advisor). The Performance of the National Industrial Segments, Composed of Publicly Traded Companies, in Light of Brazilian and World Economic Activity Level. Rio de Janeiro, 2003. 161p. MSc. Dissertation Departamento de Administração, Pontifícia Universidade Católica do Rio de Janeiro.

In pursuit of the objective of evaluating the performance of the national industrial segments, composed of publicly traded companies, in light of Brazilian and world economic activity level, the present dissertation attempts to contribute to the development of forecast models intended to aid managers and investors in the decision making process. The relationship between several levels of profit, shown as Net Sales, Gross Profit, Operating Profit and Net Profit achieved by the national industrial segments, composed of listed companies negotiated on São Paulo Stock Exchange, and the level of economic activity, represented by the national and global GDP measured through the period comprised between January 1996 and December 2002 on a quarterly basis, was studied. The first part is dedicated to analyzing the elasticity of several levels of profit of the segments evaluated in terms of the GDP variations. The second part analyses the correlation rate between the global GDP and the performance of the industrial segments. Considerable collinearity was identified between the national GDP and the global GDP, invalidating the use of these two variables simultaneously in order to evaluate the performance of the industrial segments. During the last part, an attempt was made to include other macro economic variables, in order to obtain more explanatory power of the performance of the segments in light of the variations of the level of national economic activity. Considering the statistic significance level obtained during these tests, the potential of using these relations in a forecast model was proved.

\section{Keywords}

Income Elasticity; Regression; Forecasting Models; GDP; Profits. 


\section{SUMÁRIO}

1 O PROBLEMA _ 12

1.1 Introdução __ 12

1.2 Objetivos _ _ 14

1.3 Questões a Serem Respondidas __ 16

1.4 Hipóteses 17

1.5 Delimitação do Estudo __ 22

1.6 Relevância do Estudo __ 24

2 REFERENCIAL TEÓRICO _ 26

2.1 Definição do PIB e PNB e Renda Nacional ___ 28

2.2 Estudos que Abordam a Previsão do PIB e Outras Variáveis Macroeconômicas

2.3 Estudos que Exploram as Variáveis sob o Controle das Empresas que Impactam em seus Desempenhos ___ 35

2.4 Estudos que Relacionam o Nível da Atividade Econômica e o Desempenho das Empresas e/ou Setores da Indústria

3 METODOLOGIA _ 44

3.1 Tipo de Pesquisa __ 44

3.2 Universo e Amostra __ 44

3.3 Levantamento dos Dados _ $\quad 49$

3.4 Tratamento dos Dados _

4 RESULTADOS _ 58

4.1 Análise da Correlação entre PIB e os Níveis de Lucro dos Setores ___ 58

4.2 Análise das Regressões entre os Níveis de Lucros e o PIB ___ 62

4.2.1 Análise da Receita Operacional Líquida em Função do PIB ___ 65

4.2.2 Análise do Lucro Bruto em Função do PIB _ 75

4.2.3 Análise do Lucro Operacional Próprio em Função do PIB ___ 78

4.2.4 Análise do Lucro Líquido em Função do PIB ___ 82

4.2.5 Resumo das Elasticidades dos Níveis de Lucro em Relação ao PIB__ 84 
4.3 Análise da Correlação entre GDP M, PIB e os Níveis de Lucro dos Setores _86

4.4 Análise das Séries de Lucros com a Inclusão das Variáveis Macroeconômicas Complementares

4.4.1 Análise da Receita Operacional Líquida com a Inclusão das Variáveis Macroeconômicas Complementares

4.4.2 Análise do Lucro Bruto com a Inclusão das Variáveis Macroeconômicas Complementares

4.4.3 Análise do Lucro Operacional Próprio com a Inclusão das Variáveis Macroeconômicas Complementares

4.4.4 Análise do Lucro Líquido com a Inclusão das Variáveis Macroeconômicas Complementares

4.4.5 Resumo das Elasticidades dos Níveis de Lucro em Relação ao PIB e às Demais Variáveis Macroeconômicas 99

4.5 Análise da Sazonalidade das Séries de Lucros 103

5 CONCLUSÕES 109

7 ANEXOS 123

7.1 Composição dos Setores e Empresas Sobreviventes 123

7.2 Resultados Obtidos para as Variáveis Macroeconômicas 130

7.3 Regressões da ROL X PIB 131

7.3.1 Regressões Transformadas da $\mathrm{ROL}^{*} \mathrm{X} \mathrm{PIB}^{*}$ 134

7.4 Regressões LB X PIB e Regressões Transformadas LB* X PIB* 136

7.5 Regressões LOP X PIB e Regressões Transformadas LOP* X PIB 140

7.6 Regressões LL X PIB e Regressões Transformadas LL* X PIB* 143

7.7 Regressões Stepwise para Variáveis Macroeconômicas Complementares Receita Operacional Líquida

7.8 Regressões Stepwise para Variáveis Macroeconômicas Complementares Lucro Bruto

7.9 Regressões Stepwise para Variáveis Macroeconômicas Complementares Lucro Operacional Próprio

7.10 Regressões Stepwise para Variáveis Macroeconômicas Complementares Lucro Líquido 


\section{TABELAS}

Tabela 1 - Número de empresas de capital aberto registradas na CVM. 49

Tabela 2 - Número de empresas de capital aberto listadas na Bovespa. 49

Tabela 3 - Seleção dos setores e empresas sobreviventes. 52

Tabela 4 - Empresas e setores sobreviventes e representatividade da Receita Líquida.

Tabela 5 - Indicadores macroeconômicos e respectivas fontes. 55

Tabela 6 - Variáveis macroeconômicas, formas de mensuração e unidades de medida. 55

Tabela 7 - Correlação do PIB X Níveis de Lucro dos Setores. 59 Tabela 8 - Estabilidade das relações entre ROL e PIB para dois períodos consecutivos. 64

Tabela 9 - Elasticidade da Receita Operacional Líquida em função do PIB. 70

Tabela 10 - Elasticidade da Receita Operacional Líquida em função do PIB com variáveis transformadas. 73

Tabela 11 - Elasticidade do Lucro Bruto em função do PIB. __ 76

Tabela 12 - Elasticidade do Lucro Operacional Próprio em função do PIB. ___ 81

Tabela 13 - Elasticidade do Lucro Líquido em função do PIB.

Tabela 14 - Resumo das Elasticidades dos níveis de lucro em função do PIB. ___ 84

Tabela 15 - Correlação do GDP M X Níveis de Lucro dos Setores. ___ 87

Tabela 16 - Correlação do GDP M X PIB.___ 88

Tabela 17 - Elasticidade do Lucro Operacional Próprio em função do PIB e outras variáveis macroeconômicas.

Tabela 18 - Elasticidade do Lucro Líquido em função do PIB e outras variáveis macroeconômicas.

Tabela 19 - Resumo das Elasticidades das séries de lucros em função do PIB e outras variáveis macroeconômicas.

Tabela 20 - Resumo da sazonalidade das séries de lucros dos setores da indústria nacional. 105

Tabela 21 - Resumo da sazonalidade das séries de lucros do setor de Comércio. 106 


\section{FIGURAS}

Figura 1 - Regressão Ln (Alim) x Ln (PIB). 65

Figura 2 - Análise das condições de linearidade e homoscedasticidade dos resíduos para $\operatorname{Ln}($ Alim) x Ln (PIB). 66

Figura 3 - Teste de normalidade dos resíduos da regressão logarítmica Ln (ROL Alim) $X$ $\operatorname{Ln}(\mathrm{PIB})$ 67

Figura 4 - Teste de autocorrelação de Durbin-Watson. 67

Figura 5 - Comparação entre valores observados e estimados para a ROL do setor de Alimentos e Bebidas utilizando a regressão proposta. 69

Figura 6 - Regressão logarítmica original para o setor de Petróleo e Gás. 72

Figura 7 - Regressão logarítmica transformada para o setor de Petróleo e Gás. 72

Figura 8 - Regressão linear do setor de LOP Comércio X PIB. 79

Figura 9 - Regressão stepwise Ln (ROL Alimentos) versus Ln (PIB); Ln (GDP M).___ 88

Figura 10 - Regressão múltipla Ln (ROL Alimentos) versus Ln (PIB); Ln (GDP M).__ 89

Figura 11 - Regressão stepwise para ROL do setor Mineração. 91

Figura 12 - Regressão múltipla Ln (ROL Miner) versus Ln (PIB); VJ; VC para o setor Mineração. 92

Figura 13 - Regressão múltipla transformada Ln (ROL Miner) versus Ln (PIB); VJ; VC para o setor Mineração. 93

Figura 14 - Análise das condições de linearidade e homoscedasticidade dos resíduos para Ln (Miner) versus Ln (PIB); VJ; VC. 93

Figura 15 - Teste de normalidade dos resíduos da regressão logarítmica transformada Ln (Miner) versus Ln (PIB); VJ; VC. 94

Figura 16 - Regressão múltipla Ln (LB Alim) versus Ln (PIB); VC para o setor de Alimentos e Bebidas. 96 